\title{
Performance Evaluation of Mesh - Based Multicast Routing Protocols in MANET's
}

\author{
M. Nagaratna \\ Assistant Professor \\ Dept. of CSE \\ JNTUH, Hyderabad, India
}

\author{
V. Kamakshi Prasad \\ Prof \& Additional Cont. of. \\ Examinations \\ School of Information Technology \\ JNTUH, Hyderabad, India
}

\author{
Raghavendra Rao \\ Professor \& Head \\ Dept. of CSE \\ University of Hyderabad, India
}

\begin{abstract}
Multicasting is a challenging task that facilitates group communication among the nodes using the most efficient strategy to deliver the messages over each link of the network. In spite of significant research achievements in recent years, efficient and extendable multicast routing in Mobile Ad Hoc Networks (MANETs) is still a difficult issue. This paper proposes the comparison of ODMR and PUMA protocol. As per the simulation results PUMA is better than ODMR.
\end{abstract}

Keywords- MANET; multicast; QoS.

\section{INTRODUCTION}

An ad hoc mobile network is a collection of mobile nodes that are dynamically and arbitrarily located in such a manner that the interconnections between nodes are capable of changing on a continual basis [1]. The primary goal of an ad hoc network routing protocol is to provide an efficient route establishment between a pair of nodes so that messages may be delivered in a timely manner. Route construction should be done with a minimum of overhead and bandwidth consumption. Multicasting plays an important role for communication in a MANET, where group tasks are often deployed. For multicasting, a multicast group is constructed with one or more group members and multicast address is assigned to each group. In a MANET, the group members randomly spread and frequently move in the whole network, which causes more difficulty in packet delivery and group maintenance.

Quality of service (QoS) is an important consideration in networking, but it is also a significant challenge [3, 4, 9, 11]. QoS is more difficult to guarantee in MANETs than in other type of networks, because the wireless bandwidth is shared among adjacent nodes and the network topology changes as the nodes move. With the extensive applications of MANETs in many domains, the appropriate QoS metrics should be used. Therefore, QoS multicasting routing protocols face the challenge of delivering data to destinations through multi hop routes in the presence of node movements and topology changes. According to the topology, multicast routing protocols can be classified into tree-based and mesh-based. These protocols differ in terms of the redundancy of the paths between senders and receivers. Whereas tree-based protocols provide only a single path between senders and receivers, mesh-based protocols provide multiple paths. Examples of Mesh-based protocols are ODMR and PUMA. The rest of this paper is organized as follows, section II presents about the ODMR protocol, section III presents about the PUMA protocol, section IV presents the Performance evaluation of two protocols, section V presents the simulation of NS, section VI presents simulation results of two protocols, section VII conclusions.

\section{On-Demand Multicast Routing Protocol}

Construction of a mesh it forms multiple routes and multicast packets are being delivered to destinations even the node movements and topology changes. ODMRP [2] uses the concept of forwarding group to establish a mesh for each multicast group. The forwarding group is set of nodes which are responsible for forwarding multicast data on shortest path between any member pairs. To maintain multicast group members a soft-state approach is used. Explicit control messages are not required to leave the group. ODMRP is more attractive in mobile wireless networks due to reduction of channel/storage overhead and the richer connectivity.

\section{A. Mesh Creation and multicast Route:}

This protocol establishes multicast routes and group memberships which are added to the source on- demand. If the node realizes it is in the path to the source and a segment of the forwarding group then it set the FG flag and it broadcast its own Join Reply. The Join Reply causes by every forwarding group member unless it reaches multicast source through the shortest path. In the forwarding group this process builds or adding the routes from sources to receivers and constructs a mesh. Forwarding group is set of nodes which are in charge of forwarding multicast packets and also it supports shortest paths between any member pairs. All nodes inside the multicast are members and also forwarding group nodes, forwarding group nodes forwards multicast data packets. If a multicast receiver is on the path between a multicast source and another receiver then it is said to be a forwarding group node. The mesh provides richer connectivity between multicast members as compared to multicast trees. Flooding redundancy among forwarding group helps to overcome node displacements and channel fading. Hence frequent reconfigurations are not required. 


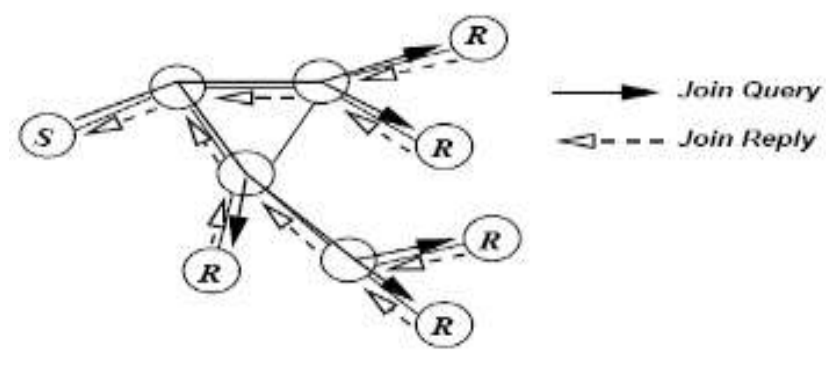

Fig 1. On-demand procedure for membership setup and maintenance

Fig 2 is an example to show the robustness of a mesh configuration. Three sources $\left(S_{1}, S_{2}\right.$, and $\left.S_{3}\right)$ send multicast data packets to three receivers $\left(R_{1}, R_{2}\right.$, and $\left.R_{3}\right)$ via three forwarding group nodes (A, B, and $\mathrm{C})$. In case the route from $S_{1}$ to $R_{2}$ is $\left\langle S_{1}-A-B-R_{2}\right\rangle$. In a tree configuration, if the link between nodes $A$ and $B$ breaks $R_{2}$ cannot receive any packets from S1 until the tree is reconfigured but in ODMRP has a redundant route $\left\langle\mathrm{S}_{1}-\mathrm{A}-\mathrm{C}-\mathrm{B}-\mathrm{R}_{2}\right\rangle$ to deliver packets without going through the broken link between nodes $\mathrm{A}$ and $\mathrm{B}$. Nodes $R_{2}$ and $R_{3}$ sends their Join Replies to both $S_{1}$ and $S_{2}$ through $I_{2}$, and $R_{1}$ sends its packet to $S_{1}$ through $I_{1}$ and $S 2$ through $I_{2}$ as shown in Fig 3.

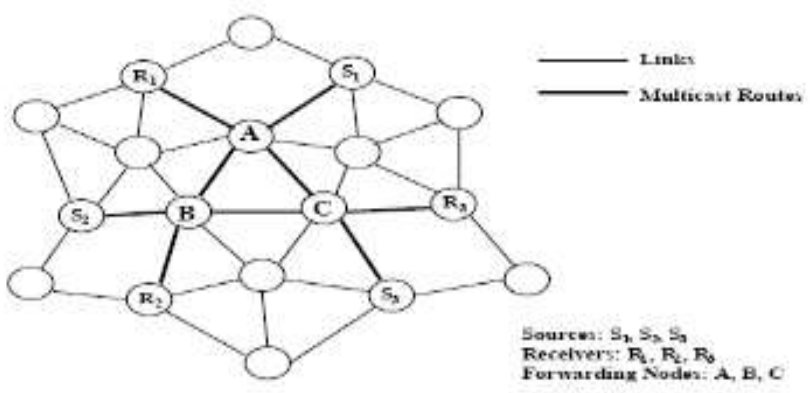

Fig 2. Mesh Configuration

When receivers send Join Replies to next hop nodes, an intermittent node $\mathrm{I}_{1}$ set the FG Flag and built its own Join Reply as there is a next node ID entry in the Join Reply received from $R_{1}$ that verifies it's ID. Note that the Join Reply built by $I_{1}$ having an entry for sender $S_{1}$ and not for $S_{2}$ as the next node ID for $S_{2}$ in the received Join Reply is not $\mathrm{I}_{1}$. At the same time node $\mathrm{I}_{2}$ set the FG Flag and it builds its own Join Reply and sends its neighbors. However $\mathrm{I}_{2}$ receives three Join Replies from the receivers, it broadcasts the Join Reply only once because the second and third table to hold no new source information. Channel overhead is
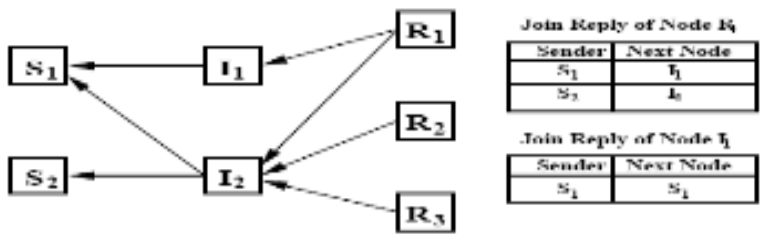

Fig 3.example of Join Reply forwarding

\section{B. Data Forwarding:}

After establishing the group and the route construction process a multicast source sends packets to receivers through selected routes and forwarding groups. Periodic control packets are delivered only when outgoing data packets are still present.
While receiving a multicast data packet a node forwards only if it is not a duplicate, set FG Flag for the multicast group which was not expired. This minimizes traffic overhead and to save sending packets through stale routes.

\section{Soft State}

In ODMRP to join or leave the group explicit control packets are need not be sent. In case a multicast source wants to leave group it immediately stops sending Join Query packets because it is not having any multicast data to sent to the group. From a specific multicast group a receiver no longer wants to receive it removes the corresponding entries from its Member Table and need not transmit the Join Reply for that group.

\section{Selection of Timer Values:}

The performance of the ODMRP is based on the timer values of route refresh interval and forwarding group timeout interval. The selection of soft state timers must be adaptive to network environment i.e. mobility pattern, capacity of the channel, type of traffic, load traffic, mobility speed etc. New route and membership information can be achieved frequently when small route refresh interval values are used at the cost of getting more packets and causes network congestion. In case where big route refresh values are selected even less control traffic is produced, nodes may not know up-to-date route information and multicast membership.

\section{E. Unicast Capability:}

The major strength of ODMRP is unicast routing capability. It can work with any unicast routing protocol and can also operates efficiently as unicast routing protocol. Therefore it need not require a separate unicast protocol. ODMRP offers the advantage of sharing the same optional software for both unicast and multicast operation reduced drastically when many multicast receivers share the same links to source.

\section{PUMA}

PUMA [6] supports any source to send multicast packets addressed to a given multicast group. PUMA does not need another unicast routing protocol because it can act as unicast protocol. PUMA implements a distributed algorithm to elect one of the receivers of a group as core of the group. The election algorithm used in PUMA is same as the spanning tree algorithm introduced by Perlman for internet works of transparent bridges [7]. Within a finite time router can find multiple paths to the core. All nodes on shortest paths between any receiver and the core collectively form the mesh.

A sender sends a data packet to the group along any of the shortest paths between the sender and the core. When the data packet reaches a mesh member, it is flooded within the mesh, and nodes maintain a packet ID cache to drop duplicate packets. PUMA uses single control message for all its functions, i.e. multicast announcement packet (MAP). Each MAP has a sequence number, group ID, core ID, distance to the core, mesh member flag, and a parent that states the preferred neighbor to reach the core. Successive MAPs' have a higher sequence number than previous multicast announcements sent by the same core. With the information contained in such announcements, nodes elect cores, determine 
the routes for sources outside a multicast group to unicast multicast data packets towards the group, notify about joining or leaving the mesh of a group and maintain the mesh.

\section{A. Connectivity Lists and propagation of Multicast Announcements:}

A node which is core of a group transmits multicast announcements periodically for that group. As the multicast announcement travels through the network, it establishes a connectivity list at every node in the network. Using connectivity lists, nodes will be able to establish a mesh, and route data packets from senders to receivers. A node stores the data from all the multicast announcements it receives from its neighbors in the connectivity list. Fresh multicast announcements overwrite entries with lower sequence numbers for the same group. For a given group, a node has only one entry in its connectivity list from a particular neighbor and it keeps only that information with the latest sequence number for a given core. Each entry in the connectivity list, it stores the multicast announcement, stores the time when it was received, and the neighbor from which it was received. Next the node generates its own multicast announcement based on the best entry in the connectivity list. For the same core ID and sequence number, multicast announcements with smaller distances to the core are considered. When all those fields are the same, the multicast announcement that arrived earlier is considered. After selecting the best multicast announcement, the node generates the fields of its own multicast announcement i.e. Core ID, Group ID, Sequence number, Distance to core, Parent, Mesh member. The connectivity list stores information about all the routes that exist to the core. When a core change occurs for a group then the node clears the entries of its old connectivity list and builds a new list, specific to the new core.

\section{B. Mesh Establishment and Maintenance:}

At the initial stage only receivers are considered as mesh members and their mesh member flag is set to TRUE in the MAP's. Non receivers consider themselves as mesh members if and only if they have at least one mesh child in their connectivity list. A neighbor in the connectivity list is a mesh child if (i) Its mesh member flag is set (ii) The distance to core of the neighbor is larger than the node's distance to core (iii) The multicast announcement corresponding to this entry was received in within a time period equal to two MAP intervals. If a node has a mesh child and is hence a mesh member, then it means that it lies on a shortest path from a receiver to the core.

\section{Core Election:}

When a new receiver wants to join a multicast group, it first finds whether it has received a MAP from core of that group. If the node has received it earlier, it adopts the core specified in the announcement it has received, and it starts transmitting MAP that specify the core for that group. It considers itself as the core of the group and starts transmitting MAP periodically to its neighbor stating itself as the core of the group and 0 distances to itself. Nodes propagate MAP based on the best multicast announcements they have received from their neighbors. A MAP with higher core ID is considered better than a multicast announcement with a lower core ID. Each connected component has only one core. If a receiver joins the group before any other receivers, it declares itself as the core of the group. If several receivers join the group concurrently, then the one with the highest ID is declared as core of the group. The election is held in the partition which does not have the old core.

\section{Forwarding Multicast Data Packets:}

The parent field of connectivity list entry corresponds to the node from which the neighbor received its best MAP. This field allows nonmembers to forward multicast packets towards the mesh of a group. A node forwards a multicast data packet it receives from its neighbor. The packets are then flooded within the mesh and group members use a packet ID cache to detect and discard packet duplicates. The routing of data packets from senders to receivers is also used to update the connectivity list. When a nonmember transmits a packet, it expects its parent to forward the packet. This serves as an implicit acknowledgment of the packet transmission. If the node does not receive an implicit acknowledgment within ACK-TIMEOUT then it deletes the parent from its respective connectivity list.

\section{Performance Evaluation}

The parameters used in calculating the performance of protocols [8,10] are Packet Delivery Ratio, Throughput, Endto-End Delay, Latency, no. of sent packets. Packet Delivery Ratio is the ratio of the data packets delivered to the destination. Throughput it is defined as the total amount of data a receiver $R$ actually receives from all the senders of the multicast group divided by the time it takes for $R$ to receive the last packet. End - to- End Delay this represents the average time it takes for a data packet to be transmitted from one forwarding node to another. Latency this represents the average time a data packet takes to travel from the transmitter to the receiver.

\section{Simulation}

The selected protocols are evaluated using Network simulator (NS-2) of 50-200 nodes incrementing by 50 nodes. Simulation runs for 100 seconds. The mobility model is selected as Random Way Point model. In this mobility model a node randomly selects a destination and it moves in the direction of the destination with a speed uniformly chosen between the minimal speed and maximal speed. After it reaches the destination, the node stays there for a pause time and then moves again. Each node moves randomly with a speed of $0-10 \mathrm{~m} / \mathrm{s}$ and stays at the same place with a pause time 0-10s. The Distributed Coordinated Function (DCF) of IEEE 802.11 for wireless LANs is assumed as the MAC layer protocol. The Two Ray Ground model is selected for the propagation. A bandwidth of $2 \mathrm{Mbps}$ with a radio range of $250 \mathrm{~m}$ is considered. 5 senders and 20 receivers were selected at random and the traffic senders send data packets of size 1460 Bytes each with a data rate of 10 packets/sec. we have chosen CBR as the type of communication and the maximum interface queue length is 250. The performance metrics considered are Throughput, Average End-to-End delay, Packet Delivery Ratio. 


\section{SimUlation RESUlts}

Simulation results of ODMR and PUMA protocol for varying the node mobility and their group sizes.

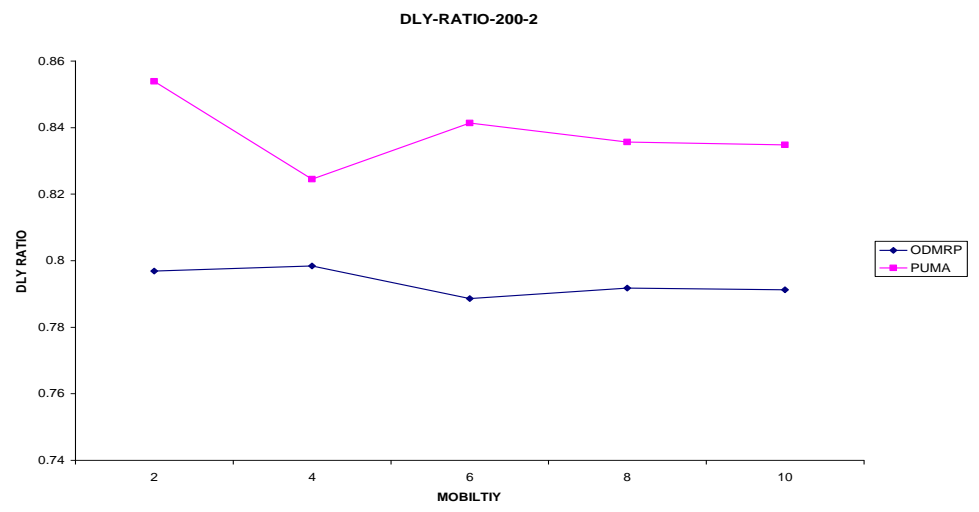

Fig 4. Packet delivery Ratio for 200 nodes and their group size is two

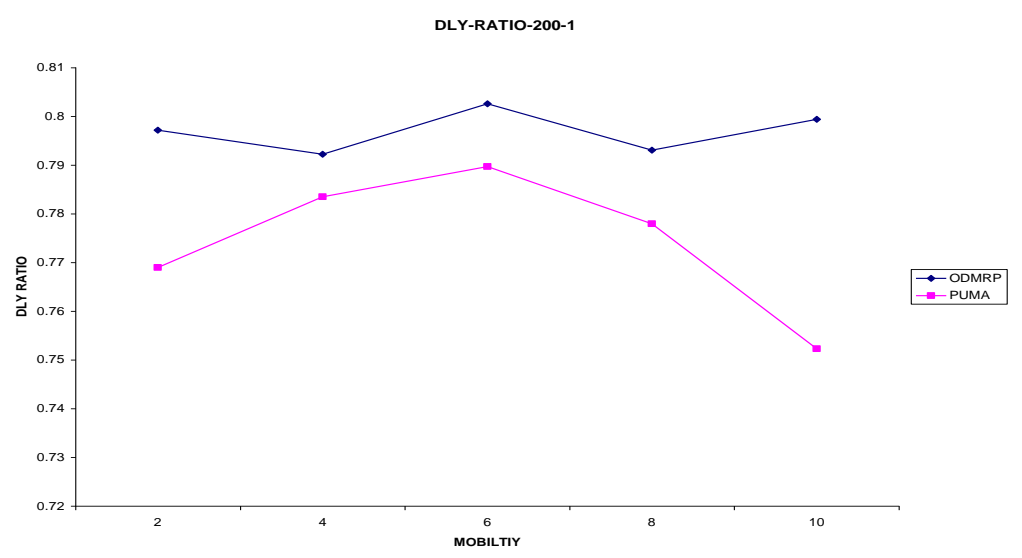

Fig 5. Packet delivery Ratio for 200 nodes and their group size is one

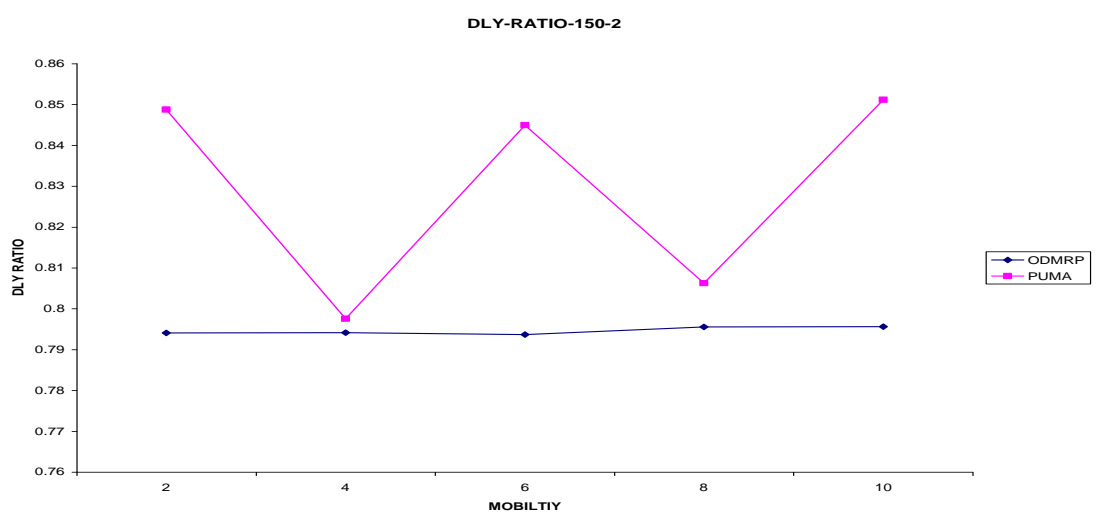

Fig 6. Packet delivery Ratio for 150 nodes and their group size is two 


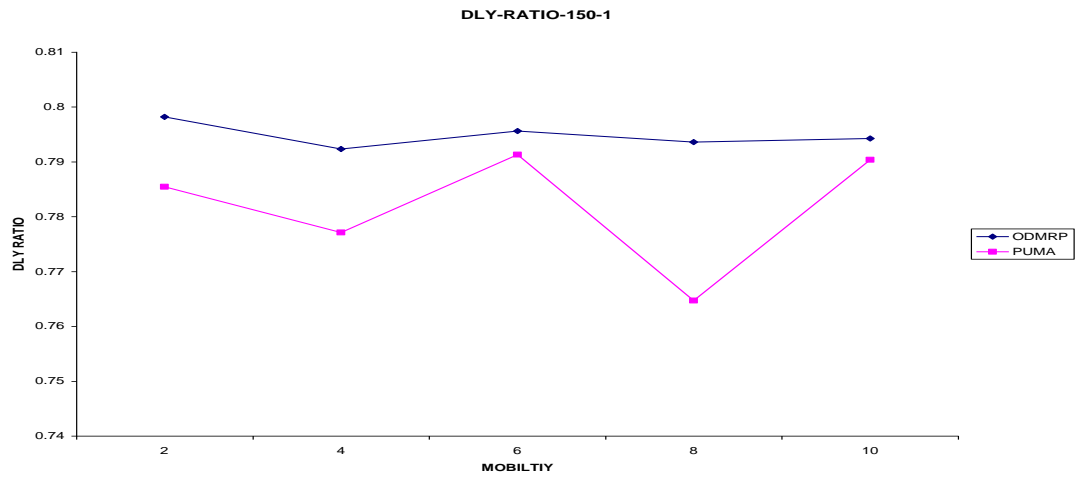

Fig 7. Packet delivery Ratio for 150 nodes and their group size is one

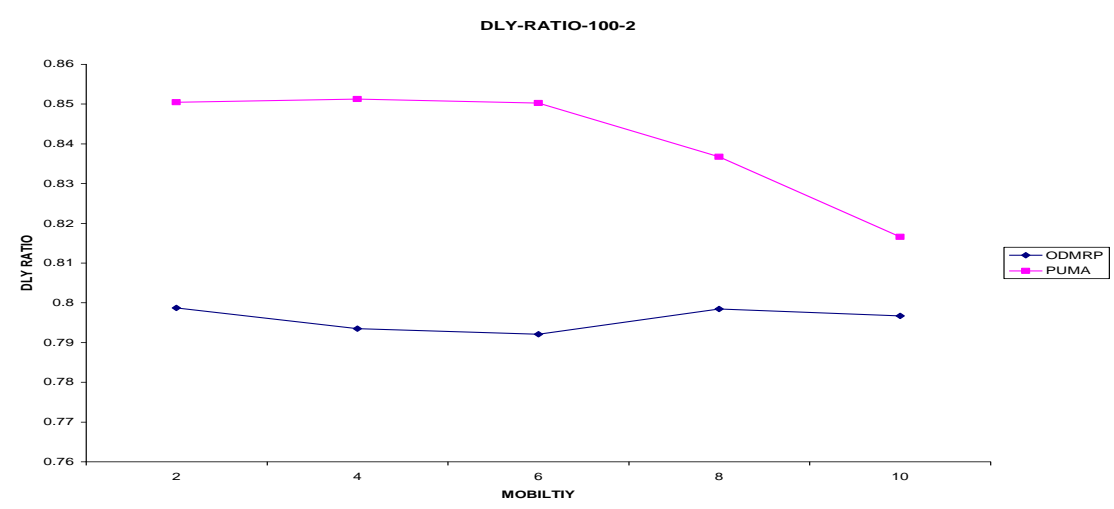

Fig 8. Packet delivery Ratio for 100 nodes and their group size is two

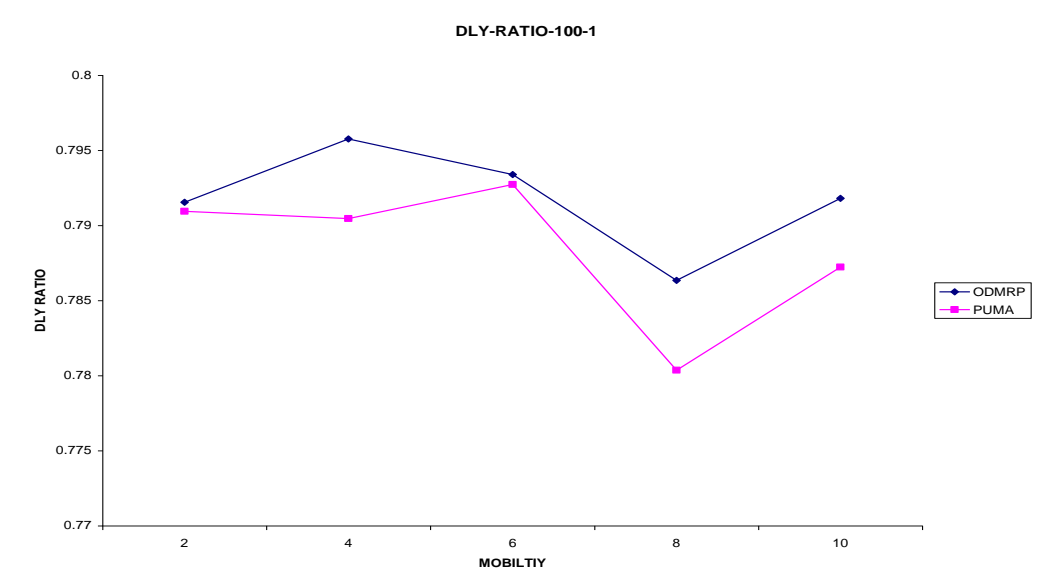

Fig 9. Packet delivery Ratio for 100 nodes and their group size is one 


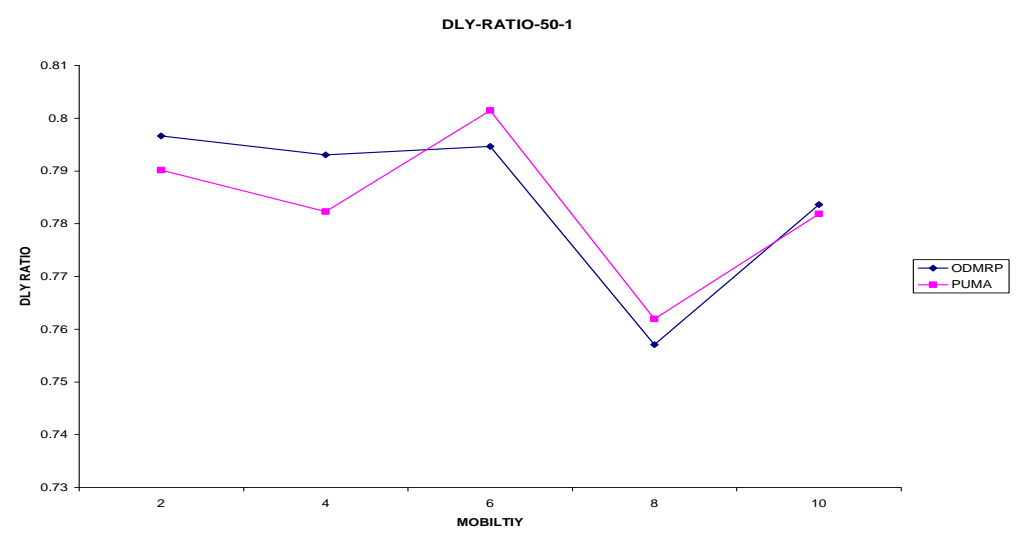

Fig 10. Packet delivery Ratio for 50 nodes and their group size is one

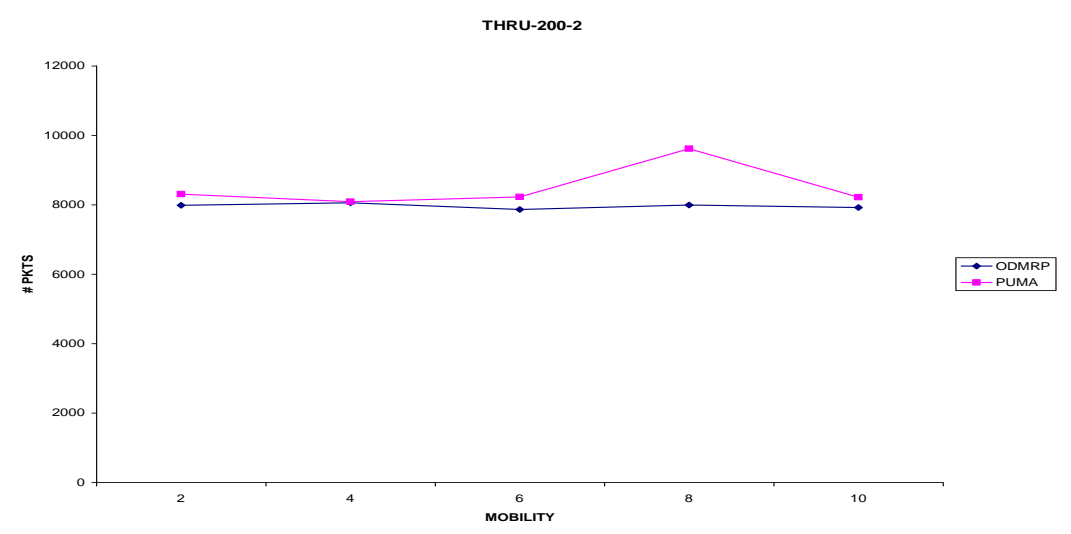

Fig 11. Throughput for 200 nodes and their group size is two

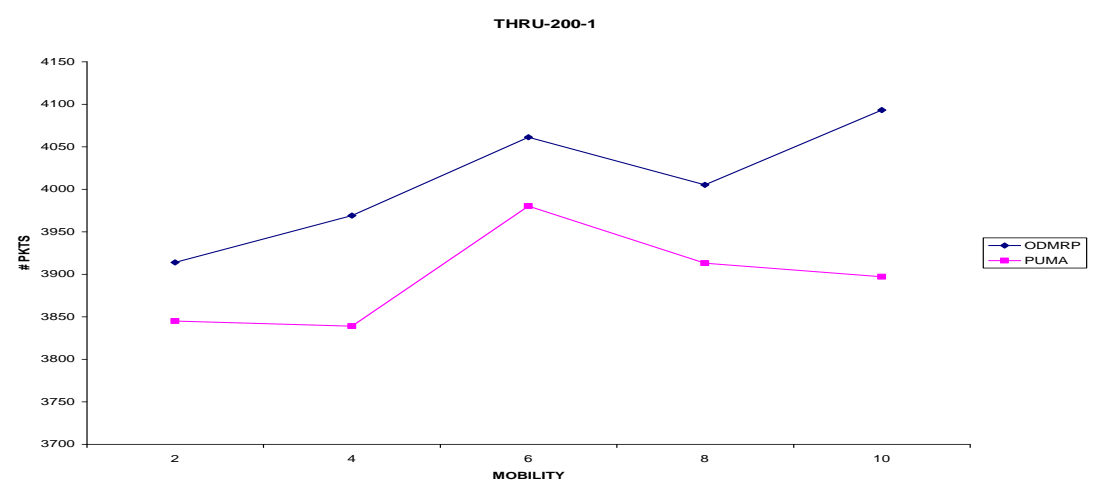

Fig 12. Throughput for 200 nodes and their group size is one 


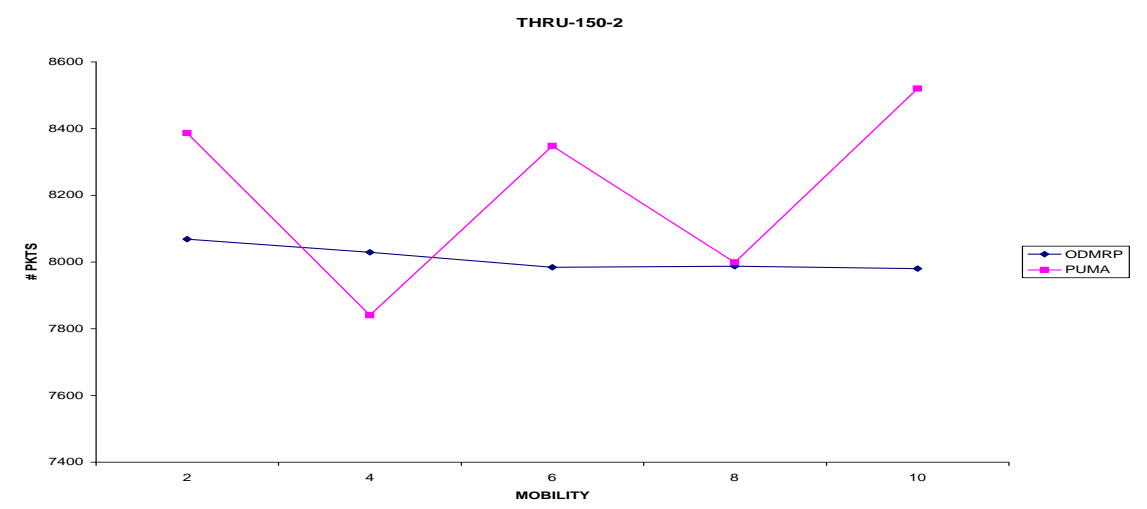

Fig 13. Throughput for 150 nodes and their group size is two

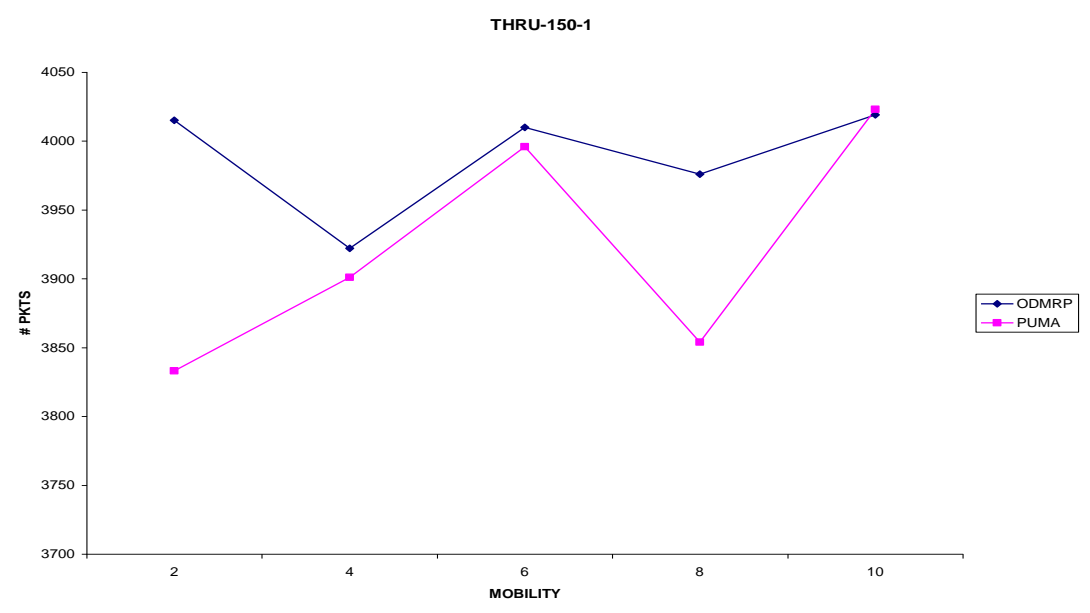

Fig 14. Throughput for 150 nodes and their group size is one

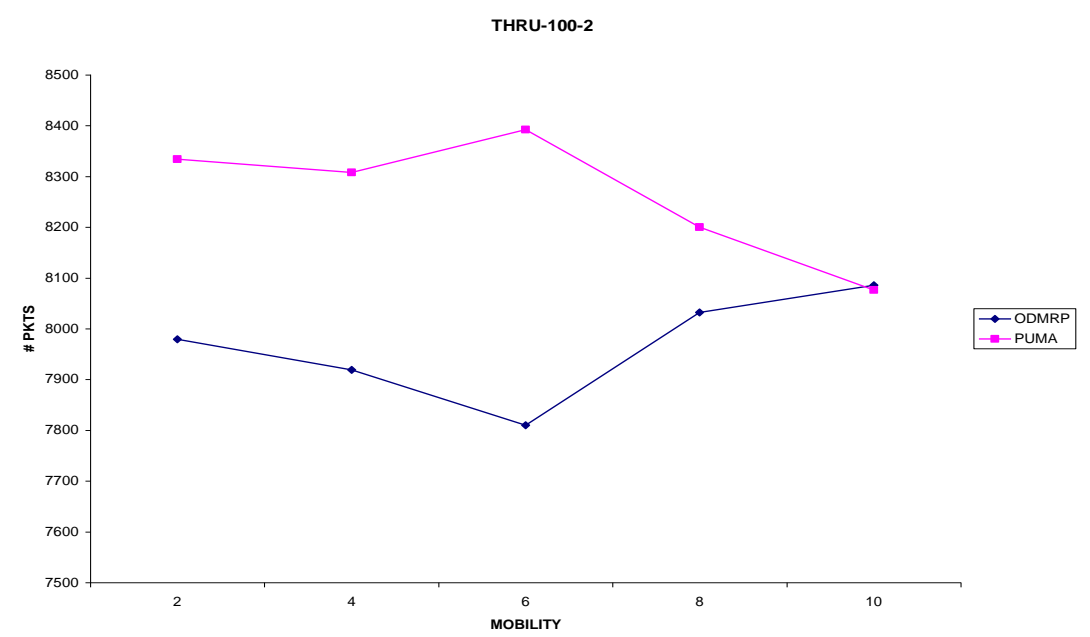

Fig 15. Throughput for 100 nodes and their group size is two 


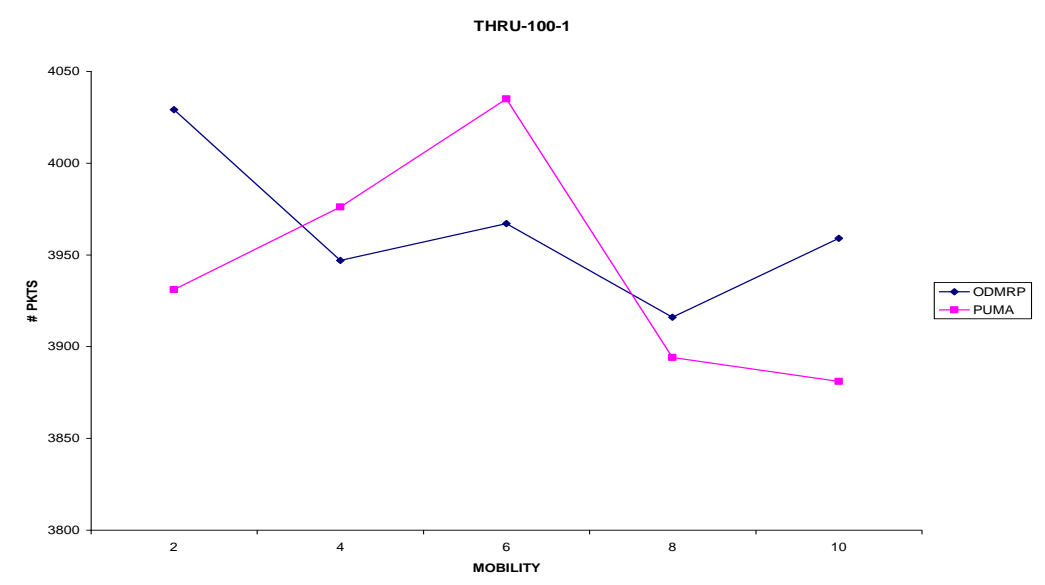

Fig 16. Throughput for 100 nodes and their group size is one

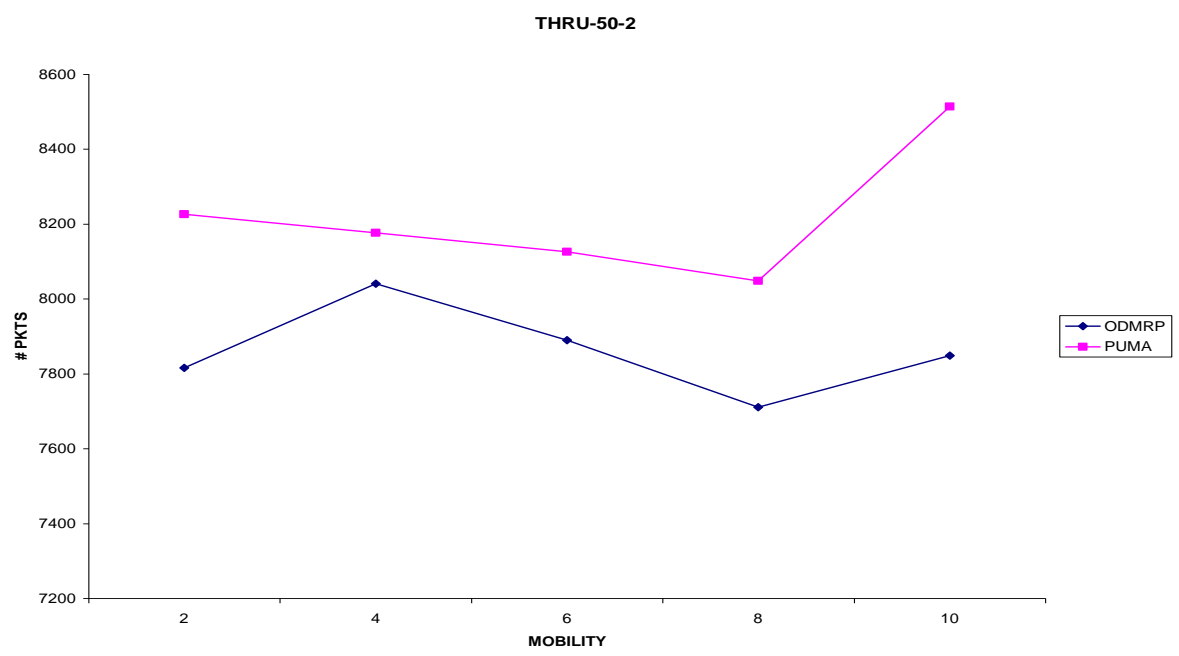

Fig 12. Throughput for 50 nodes and their group size is two

THRU-50-1

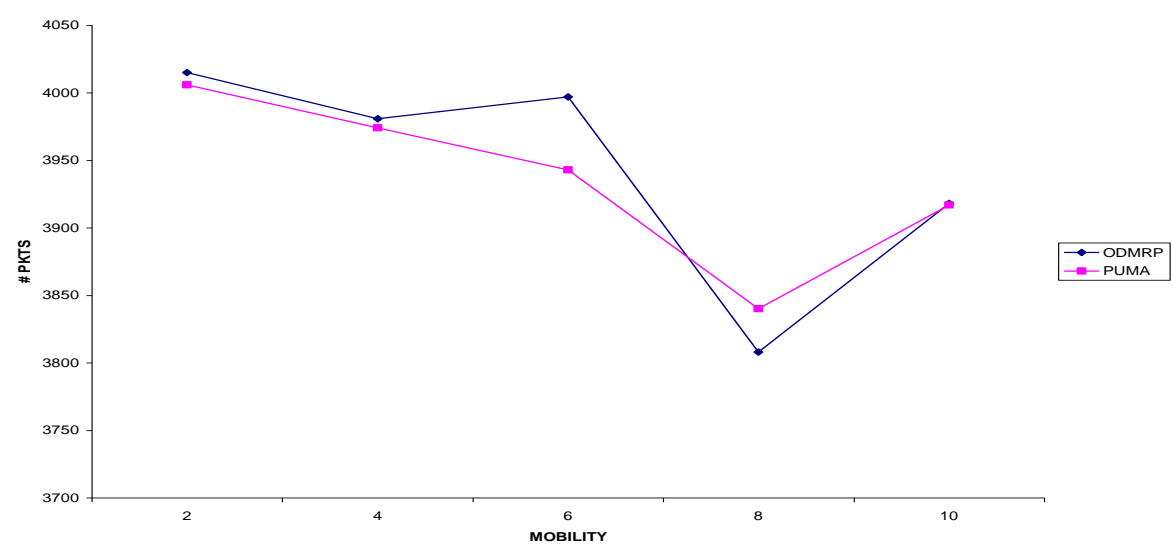

Fig 12. Throughput for 50 nodes and their group size is one

\section{CONCLUSIONS}

This paper proposes the comparison of ODMR and PUMA protocol. As per the simulation results PUMA improves the performance of ODMR. PUMA improves the throughput, packet delivery ratio by varying the node mobility and their group sizes.

\section{REFERENCES}


[1] G. Santhi and Dr. A. Nachiappan , "Agent Based Adaptive Multicast Routing with QoS guarantees in MANETs", 2010 Second International conference on Computing, Communication and Networking Technologies.

[2] S. J. Lee, W. Su, and M. Gerla, "On-demand multicast routing protocol in multihop wireless mobile networks," ACM/Kluwer Mobile Networks and Applications, Vol. 7, No. 6, 2002, pp. 441-453.

[3] H. Chen, B. L. Sun, and Y. Zeng, "QoS Multicast Routing Algorithm in MANET: An Entropy-Based GA," Lecture Notes in Artificial Intelligence, Vol. 4114, Springer Verlag, 2006, pp. 1279-1289.

[4] C. R. Lin, "On-demand QoS Routing in Multihop Mobile Networks," In: Proceedings of IEEE INFOCOM 2001, April 2001, pp. 1735-1744.

[5] The Network Simulator NS-2: http://www.isi.edu/ns-nam/ns/.

[6] Ravindra Vaishampayan, J.J. Garcia-Luna-Aceves

"Efficient and Robust Multicast Routing in Mobile Ad Hoc Networks", 2004 IEEE International Conference on Mobile Ad-hoc and Sensor Systems.
[7] R. Pedman, "An algorithm for distributed computation of a spanning tree in an extended Ian:' in ACM Special Interest Group on Data Comrnunication (S1GCOMM). 1985, pp. -53.

[8] S.-J. Lee, W. Su, J. Hsu, M. Gerla and R. Bagrodia. A Performance Comparison Study of Ad-Hoc Wireless Multicast Protocols. In Proceedings of IEEE INFOCOM 2000, pages 565-574, March 2000.

[9] Qi Xue, Aura Ganz, Ad Hoc QoS On-demand Routing (AQOR) In Mobile Ad Hoc Networks, Journal of Parallel and Distributed Computing, Volume 63, Issue 2, February 2003, pp.: 154-165.

[10] S. Corson, J. Macker, Mobile Ad hoc Networking (MANET): Routing Protocol Performance Issues and Evaluation Considerations, RFC2501.

[11] G.I. Ivascu, S.Pierre and A. Quintero, QoS Support based on a Mobile Routing Backbone for Ad Hoc Wireless Networks, IWCMC'06, July 3 6, 2006, Vancouver, Canada, pp.: 121-126. 